\title{
Animal Models of Glaucoma
}

\author{
Rachida A. Bouhenni, ${ }^{1}$ Jeffrey Dunmire, ${ }^{1}$ Abby Sewell,, ${ }^{1}$ and Deepak P. Edward ${ }^{2,3}$ \\ ${ }^{1}$ Department of Ophthalmology, Summa Health System, Akron, OH 44304, USA \\ ${ }^{2}$ Wilmer Eye Institute, Johns Hopkins University, Baltimore, MD 21210, USA \\ ${ }^{3}$ Research Department, King Khaled Eye Specialist Hospital, P.O. Box 7191, Riyadh 11462, Saudi Arabia
}

Correspondence should be addressed to Deepak P. Edward, deepak.edward@gmail.com

Received 16 January 2012; Revised 27 February 2012; Accepted 29 February 2012

Academic Editor: Monica Fedele

Copyright $\odot 2012$ Rachida A. Bouhenni et al. This is an open access article distributed under the Creative Commons Attribution License, which permits unrestricted use, distribution, and reproduction in any medium, provided the original work is properly cited.

Glaucoma is a heterogeneous group of disorders that progressively lead to blindness due to loss of retinal ganglion cells and damage to the optic nerve. It is a leading cause of blindness and visual impairment worldwide. Although research in the field of glaucoma is substantial, the pathophysiologic mechanisms causing the disease are not completely understood. A wide variety of animal models have been used to study glaucoma. These include monkeys, dogs, cats, rodents, and several other species. Although these models have provided valuable information about the disease, there is still no ideal model for studying glaucoma due to its complexity. In this paper we present a summary of most of the animal models that have been developed and used for the study of the different types of glaucoma, the strengths and limitations associated with each species use, and some potential criteria to develop a suitable model.

\section{Introduction}

Glaucoma is a leading cause of blindness and visual impairment worldwide affecting 70 million people [1]. It is a devastating disorder that leads to retinal ganglion cell (RGC) degeneration, visual field loss, and, eventually, blindness. To date, over 3 million Americans suffer from glaucoma, with another 100,000 patients being diagnosed each year $[1,2]$. Although efforts and research in the field of glaucoma are substantial, its pathophysiology is not completely understood.

Animal models have greatly improved our understanding of the causes and progression of human diseases and have proven to be a useful tool for discovering targets for therapeutic drugs. However, several diseases remain incurable because not all models used for studying these diseases mimicked the human disorders completely.

In glaucoma, a wide variety of animal models of different species have been used to study the disease $[3,4]$. These included large animals such as monkeys [5], dogs and cats $[6,7]$, pigs [8], and small animals such as rodents [9]. Glaucoma in these animals was either spontaneous or induced.
Although these models have provided valuable information about the disease, they all had drawbacks and glaucoma remains incurable.

Several types of glaucoma have been described. These have been broadly classified as acute and chronic, secondary and primary. In general, glaucoma in humans is classified into three major types: Primary Open Angle Glaucoma (POAG), Primary Angle Closure Glaucoma (PACG), and Primary Congenital Glaucoma (PCG) with POAG being the most common type in most populations [10]. Although the final common pathway of tissue damage in all types of glaucoma is the axonal damage that manifests as optic nerve $(\mathrm{ON})$ atrophy, causing progressive visual field defects that eventually lead to blindness, each type of glaucoma may be caused by a different mechanism. Elevated intraocular pressure (IOP) is a common thread that connects most forms of glaucoma and is a major risk factor for the disease. In this paper, we describe a wide variety of the animal species that have been developed and used to study the different types of glaucoma and outline their features, unique strengths and limitations, as well as some potential criteria to develop a suitable model. 
TABLE 1: Summary of the animal models commonly used for glaucoma research.

\begin{tabular}{|c|c|c|c|}
\hline Glaucoma type & Animal & Model mode, mechanism & Reference \\
\hline \multirow{16}{*}{ POAG } & \multirow{4}{*}{ Monkey } & Spontaneous inheritance & {$[11]$} \\
\hline & & Laser photocoagulation of entire TM, reduced outflow by PAS & {$[12-17]$} \\
\hline & & Intracameral injection of latex microspheres, TM blockage & {$[18]$} \\
\hline & & Intracameral injection of autologous fixed red blood cells, TM blockage & {$[19,20]$} \\
\hline & Dog & Spontaneous inheritance & {$[21,22]$} \\
\hline & \multirow{2}{*}{ Mouse } & Transgenic, Myoc mutation & {$[23,24]$} \\
\hline & & Transgenic, alpha-1 subunit of collagen type I & {$[25,26]$} \\
\hline & Rat & Topical application of dexamethasone & {$[27]$} \\
\hline & \multirow{3}{*}{ Zebrafish } & Transgenic, bug eye mutant & {$[28,29]$} \\
\hline & & Transgenic, $\operatorname{Irp} 2$ mutation & {$[30]$} \\
\hline & & Transgenic, $w d r 36$ mutation & {$[31]$} \\
\hline & \multirow{2}{*}{ Rabbit } & Subconjunctival injection of betamethasone & {$[32-34]$} \\
\hline & & Posterior chamber injection of $\alpha$-chymotrypsin, TM blockage & {$[35-37]$} \\
\hline & Sheep & Topical application of prednisolone & {$[38-40]$} \\
\hline & Cow & Topical application of prednisolone & {$[41,42]$} \\
\hline & Birds & Light-induced, reduced outflow facility & {$[43-45]$} \\
\hline \multirow{12}{*}{ PACG } & Dog & Spontaneous inheritance & {$[46-53]$} \\
\hline & Turkey & Spontaneous inheritance & {$[54]$} \\
\hline & \multirow{5}{*}{ Rat } & Episcleral vein injection of saline, obstruction of outflow & {$[55]$} \\
\hline & & Injection of polystyrene microbeads or hyaluronic acid, TM blockage & {$[56,57]$} \\
\hline & & Cauterization of episcleral veins, reduced outflow by PAS & {$[58]$} \\
\hline & & Ligation of episcleral veins, obstruction of outflow & {$[59]$} \\
\hline & & Laser photocoagulation of translimbal region, reduced outflow by PAS & {$[60]$} \\
\hline & \multirow{3}{*}{ Mouse } & Transgenic, Vav2/Vav3 knockout & {$[61]$} \\
\hline & & Laser photocoagulation of episcleral veins, reduced outflow by PAS & {$[62,63]$} \\
\hline & & Cauterization of episcleral veins, reduced outflow by PAS & {$[64]$} \\
\hline & \multirow{2}{*}{ Rabbit } & Water loading, decreased outflow facility & {$[65,66]$} \\
\hline & & Laser photocoagulation of TM, obstruction of outflow & {$[67-69]$} \\
\hline \multirow{7}{*}{ PCG } & Rabbit & Spontaneous inheritance & {$[70-75]$} \\
\hline & \multirow{2}{*}{ Rat } & Spontaneous inheritance, WAG strain & {$[76,77]$} \\
\hline & & Spontaneous inheritance, RCS strain & {$[78,79]$} \\
\hline & Cat & Spontaneous inheritance & {$[6,21,80,81]$} \\
\hline & \multirow{2}{*}{ Mouse } & Transgenic, Cyp $1 b 1$ mutation & {$[82-84]$} \\
\hline & & Transgenic, Cyp $1 b 1$ and Tyr mutations & {$[85]$} \\
\hline & Quail & Spontaneous inheritance, al mutant & {$[86,87]$} \\
\hline Normal tension & Mouse & Transgenic, Glast or Eaac1 mutation & {$[88]$} \\
\hline Autoimmune & Rat & Immunization against HSP27 and HSP60, RGC loss & {$[89,90]$} \\
\hline Pigmentary & Mouse & DBA/2J strain, Gpnmb, and Tyrp1 mutation & [91-93] \\
\hline
\end{tabular}

TM: trabecular meshwork; PAS: peripheral anterior synechiae; RGC: retinal ganglion cell.

1.1. Primary Open Angle Glaucoma (POAG). POAG is the most common form of glaucoma in most populations. More than 20 genetic loci have been reported for POAG but only three causative genes have been identified to date (Myocilin, Optineurin, and WDR36) [94]. POAG is characterized by elevated IOP and acquired loss of RGCs and atrophy of the ON [10]. Animal models (spontaneous and induced) that have been used to study POAG (Table 1), and provided valuable information about the disease are described below.

1.1.1. Monkeys. Glaucoma in monkeys was first described in 1993, when a group of rhesus monkeys at the Cayo Santiago monkey colony in Puerto Rico examined for potential diseases in the posterior segment of the eye were found to have both low and high ( $>$ or $=22 \mathrm{mmHg}$ ) tension POAG [11]. POAG in the rhesus monkey was found to be of maternal inheritance in more than $40 \%$ of the animals demonstrating increased IOP. Affected animals exhibit a loss of RGCs, excavation of the $\mathrm{ON}$, and electrophysiological evidence of damage to the retinal peripheral field.

Experimental monkey models have also been developed for the study of POAG. Gaasterland and Kupfer developed an experimental monkey model using argon laser photocoagulation [12]. They used a modified Koeppe-type 
goniolens to laser the entire circumference of the trabecular meshwork (TM) which resulted in IOP elevation in $70 \%$ of the animals. The IOP range was between 24 and $50 \mathrm{mmHg}$ after the 4th treatment and remained elevated for 25 days. Histopathologic specimens from eyes with elevated IOP and ON cupping showed selective loss of RGCs and thinning of the nerve fiber layer compared with specimens from untreated controls suggesting that glaucoma was achieved. Several studies after that used the monkey model to describe the functional and anatomic changes that occur within the eye and $\mathrm{ON}$ in an effort to understand the reasons that lead to elevated IOP [13-17]. Other experimental monkey models of chronic IOP elevation were developed by Weber and Zelenak using latex microspheres [18] and Quigley and Addicks using autologous fixed red blood cells $[19,20]$. Another model that develops acute elevation of IOP was also used to study the mechanism of ON damage [95].

The close phylogeny and high homology of the monkey with humans makes it an excellent model for studying glaucoma. Monkeys have retinal and $\mathrm{ON}$ anatomy that is almost identical to humans. Unfortunately, monkeys are very expensive, their availability is limited, and they are difficult to handle. Experiments using monkeys require highly experienced teams and special housing facilities, making them beyond the reach of many research laboratories.

1.1.2. Dogs. In 1981, Gelatt et al. described an inherited POAG in the beagles bred in their laboratory [21]. The condition appeared to be autosomal recessive. Elevation of IOP (30 to $40 \mathrm{mmHg}$ ) in this model developed bilaterally at 1-2 years of age, tonographic recordings, and constant pressure perfusions indicated a reduction in the aqueous humor outflow. Gonioscopically, the disease had two phases: open iridocorneal angle during the onset and the first 2-4 years of the disease and closed iridocorneal angles associated with lens subluxation and displacement from the anterior vitreous patellar fossa. The animal also exhibited cupping and atrophy of the optic disc, buphthalmia, cataract formation, vitreous syneresis, and eventually phthisis bulbi. This model was recently used in a genome wide SNP array study to map the disease genes and led to the identification of the metalloproteinase ADAMTS10 as a candidate gene for POAG [22].

The advantage of using this model and dogs in general in glaucoma research is the spontaneous inheritance of the disease without congenital anomalies and the availability of the genome sequence. Dogs have relatively large eyes but can be aggressive and difficult to handle in the laboratory. Also, anatomically, dogs have an intrascleral plexus, rather than a Schlemm's canal; this difference may be minor but can be a limitation and their availability may be limited.

1.1.3. Mice. A mouse strain expressing the Tyr423His myocilin point mutation corresponding to the human MYOC Tyr437His mutation was developed to study POAG $[23,24]$. Myocilin is one of the causative genes of POAG in humans [96] and has been extensively studied. At 18 months of age, the myocilin model demonstrated loss of $\sim 20 \%$ of the RGCs in the peripheral retina, axonal degeneration in the $\mathrm{ON}$, detachment of the endothelial cells of the trabecular meshwork (TM), and moderate and persistent elevation of IOP (2 mmHg higher than normal) [23].

Another transgenic mouse strain with a targeted mutation in the gene for the $\alpha 1$ subunit of collagen type I has also been developed to study POAG. This model demonstrated open angles, progressive ON axonal loss, and gradual elevation of IOP suggesting an association between IOP regulation and fibrillar collagen turnover $[25,26]$.

There are several advantages of using mice in glaucoma research. These include the high degree of conservation between mice and human genomes, enabling genetic manipulation by altering the mouse genome, and the ability to breed the animals as desired. In addition, they are inexpensive and easy to house and handle, their eyes are easy to obtain, and the sample number for studies can be large. The disadvantages of the mouse model in glaucoma are the absence of the lamina cribrosa in the ON, the very small size of the globe which makes it hard to access clinically, and the availability of specific models may be limited.

1.1.4. Rats. A glaucoma rat model, induced by topical application of dexamethasone, was also developed to study the expression of myocilin. Although IOP was elevated after 2 weeks of treatment, the protein and mRNA levels of myocilin in the TM and around Schlemm's canal in the treated eyes were not different from those of the controls suggesting that myocilin may not be directly linked to ocular hypertension [27].

Similar to mice, rats have many advantages. In contrast to other nonprimate models, the rat shares similar anatomical $[97,98]$ and developmental $[99,100]$ characteristics of the anterior chamber, especially in the aqueous outflow pathway, with the human. Therefore, results obtained from the rat are expected to mimic changes that occur in the human. In addition, there is reasonable IOP elevation as retinal and ON changes are similar to those seen in humans. Also, reduction of IOP in response to glaucoma medications has been described but the medication effects were not all identical to those observed in humans [101]. Furthermore, rats are easier to maintain in the laboratory and similar to mice they enable genetic manipulation and can be used in large numbers.

1.1.5. Zebrafish. Transgenic teleost Danio rerio (zebrafish) models have been developed for studying glaucoma [102, 103]. The bug eye mutant that was developed by Simon et al. shows RGC death and high IOP [28, 29]. The mutant develops buphthalmia shortly after sexual maturation and an average IOP of $32.9 \pm 16.2 \mathrm{mmHg}$ compared to that in the wild type $(14.7 \pm 3.6 \mathrm{mmHg})$. This model was recently used in a study that led to the identification of a mutation in the lowdensity lipoprotein receptor-related protein 2 ( $\operatorname{Lrp} 2)$ that is important for myopia and other risk factors for glaucoma [30]. The $\operatorname{lrp} 2$ mutant exhibited a phenotype that included high IOP, enlarged eyes, decreased retinal neurons, activation of RCG stress genes, and ON pathology. Another zebrafish glaucoma model, the wdr36 mutant that was developed by Skarie and Link, was used to characterize the $w d r 36$ 
function [31]. This model, however, was only developed to study the function of $w d r 36$, as it did not show a typical glaucoma phenotype [31].

The zebrafish model has received attention for its usefulness in studying glaucoma and other human diseases $[104,105]$ because of its short generation times and a wellsupported genomic infrastructure. It allows the combination of forward and reverse genetic approaches in order to identify critical genetic interactions required for normal and pathological events. This model would be ideal for studying developmental changes in glaucoma such as those occurring in PCG. It is easily adapted to laboratory settings and can be maintained in a relatively small space. The fish typically reaches sexual maturity in 3 to 4 months, and a breeding pair can produce more than 200 fertilized eggs per mating. Fertilization is external, and the egg and embryo are transparent, which makes it easy to visualize the changes with a regular dissecting microscope. The fish develops quickly, and all major organ systems are formed by $24 \mathrm{hrs}$ after fertilization. Mutagenesis in zebrafish is performed by gamma ray and chemical approaches. The fish also enables haploid screens and diploidization, transgenesis, and forward and reverse genetic approaches which make it an attractive model for genetic manipulations of the visual system.

1.1.6. Other POAG Models. Administration of glucocorticosteroids can lead to the development of ocular hypertension and POAG through a reduction in aqueous humor outflow [106, 107]. Models using steroid-induced ocular hypertension have been developed in many animals such as rabbits, bovine, and sheep [32-34, 38-42]. A topical application of prednisolone acetate induced IOP elevation in $100 \%$ of bovine and sheep (from $16-17 \mathrm{mmHg}$ to 30 $35 \mathrm{mmHg}$ and from $11.2 \mathrm{mmHg}$ in to $23.2 \mathrm{mmHg}$ in bovine and sheep, resp.). IOP in these animals returned to normal when the treatment was discontinued. In rabbits, injection of betamethasone subconjunctivally or $\alpha$-chymotrypsin into the posterior chamber also resulted in elevated IOP that lasted for 7 weeks [35-37]. The consistency and robustness of the IOP response and the low cost of maintaining the animals developed using steroids (rats, rabbit, sheep, and cows) compared to primates are all advantages of this model. However, the prolonged topical corticosteroid treatment required to achieve glaucoma can cause significant adverse effects such as cataracts and corneal ulcers.

Avians. Light induced avian models of POAG have also been described [43, 44]. IOP in these models appeared to be responsive to several antiglaucoma drugs [45]. At 8-9 weeks of age, the chicks had significantly enlarged eyes and an IOP that was slightly lower $(13.79$ v. $16.46 \mathrm{mmHg} ; P<0.05)$. At this age, the aqueous outflow was markedly reduced but no change in aqueous inflow could be demonstrated. By 18 to 20 weeks the glaucomatous eyes were further enlarged and the IOP was higher (mean IOP 29.85 v. $22.27 \mathrm{mmHg}$; $P<0.05)$. Birds may be easy to handle in the laboratory and are not expensive. This model could be potentially valuable for studying the effect of glaucoma medications on IOP.
1.2. Primary Angle Closure Glaucoma (PACG). Similar to POAG, PACG is characterized by elevated IOP, damage to the $\mathrm{ON}$, and visual field loss. The iris in PACG obstructs the TM, whereas in POAG the TM is open and unobstructed [108]. There are several animal models that have been developed for the study of PACG (Table 1), some of these are congenital such as dogs and turkeys, and some are induced such as mice and rats.

1.2.1. Dogs. Glaucoma in dogs has been identified in Beagles, Cockers, Wirehaired Fox Terrier, Sealyham Terriers, and Basset Hounds [46-51] and was described in the late 1960s by veterinary ophthalmologists. Glaucoma in most species of dogs is of the closed angle type. Dogs may also have congenital, primary, or secondary glaucoma [52]. It is a rare condition and is caused by abnormalities in the aqueous humor outflow pathways and mimics congenital glaucoma in humans. Puppies generally present young (3-6 months of age) with an acute onset of buphthalmia and corneal edema with IOP reaching about $40 \mathrm{mmHg}$ at 18 months. It may be unilateral and bilateral and may be associated with other ocular anomalies [53]. Since the disease is rare in dogs and the genotype and phenotype of glaucoma have not been well characterized, this model has not been used to study angle closure or congenital glaucoma.

1.2.2. Turkeys. An inherited eye disease leading to secondary angle closure glaucoma was also described in a slate line of domestic turkeys (Meleagris gallopavo) [54]. The disease was progressive and the model demonstrated buphthalmia, low-grade aqueous cell, and flare associated with progressive posterior synechiae formation resulting in papillary block and iris bombe. IOP in this model was significantly increased and was associated with an increase in corneal diameter. This model is good for studying angle closure glaucoma; however, its availability may be limited.

1.2.3. Mice. Genetically manipulated Vav2/Vav3-deficient mice were also described and found to have elevated IOP, which eventually manifests as buphthalmos [61]. Loss of Vav2 and Vav3 expression in these mice is associated with changes in the iridocorneal angle, which leads to chronic angle closure. The elevation of IOP is accompanied by selective loss of RGCs and optic nerve head (ONH) excavation. The characteristics that make this model useful for glaucoma research are as follows: (1) the elevated IOP occurs spontaneously in these mice and does not require the ocular manipulation necessary in induced models, (2) the frequency of the ocular phenotype is high and onset occurs at a relatively young age, and (3) ocular hypotensives commonly used to treat human glaucoma show efficacy in lowering IOP in this model. The most significant advantage of this mouse glaucoma model is that the deleted genes, Vav2 and Vav3, are well-focused targets that have been studied for over 20 years, providing a useful starting point for further investigation of the potential molecular mechanisms underlying this phenotype. 
1.2.4. Additional PACG Models. A wide variety of rat and mouse models have been developed to study the effect of elevated IOP on the ON and RGC degeneration. Though these models were primarily developed to study retinal IOP-related posterior segment damage, the histopathological examination showed varying degrees of angle closure. IOP elevation has been induced by a number of techniques that include the use of hypertonic saline injection into the episcleral veins, cauterization or ligation of episcleral veins, or laser photocoagulation of the perilimbal region.

(1) Rats. Episcleral vein saline injections of Brown Norway rats resulted in sustained IOP elevations after 4 weeks in $45 \%$ of rats with $35 \%$ developing sustained elevations after subsequent injections [55]. Of those having sustained elevations, the mean IOP change from baseline ranged from 7 to $28 \mathrm{mmHg}$. IOP change of 10 to $20 \mathrm{mmHg}$ for more than 3 weeks or greater than $20 \mathrm{mmHg}$ for over 1 week resulted in total involvement of the $\mathrm{ON}$ with occasional axons that appeared morphologically normal. Electron micrographs of eyes from this model showed axons within damaged nerves which were frequently swollen associated with accumulation of vesicles, dense bodies, and swollen mitochondria providing histological evidence of glaucoma. Additional models using fluorescent polystyrene microbeads and hyaluronic acid injections have also been developed [56, 57]. These models showed a significant IOP elevation and glaucomatous damage in the retina. Wistar rats injected with a solution of microbeads demonstrated an IOP of $29.7 \mathrm{mmHg}$ that remained stable for 13 days and resulted in an axon density that was $16 \%$ lower than that in the control groups [56]. Wistar rats receiving weekly injections of hyaluronic acid had elevated IOP in the low $20 \mathrm{~s}$ for the duration of the 10 weeks. Eyes enucleated after 10 weeks showed significant loss of RGCs [57].

When cauterization of episcleral veins of Wistar rats was used [58], an IOP elevation from $13.2 \mathrm{mmHg}$ to $53 \mathrm{mmHg}$ was noted. There was an increase of 2.3 -fold above the mean normal IOP at 2.5 months, whereas ligation of the episcleral veins increased IOP from $20.2 \mathrm{mmHg}$ to $27.7 \mathrm{mmHg}$ after one week and IOP elevation persisted for 7 months in $40.8 \%$ of animals [59]. Further ligation was needed in $59.2 \%$ of animals for induced persistent IOP elevation. At 24 weeks there was a $35 \%$ reduction in the RGC number compared with control retinas. Intracameral India Ink injection resulted in a dark circle along the circumference of the limbus. Translimbal photocoagulation of the darkened area raised IOP to greater than $25 \mathrm{mmHg}$ after 3 laser treatments; however, further laser treatments were necessary to maintain IOP > $20 \mathrm{mmHg}$ during the course of the study. The thickness of the nerve fiber layer decreased in the glaucomatous eyes and the surface nerve fiber layer and prelaminar region of the $\mathrm{ON}$ were considerably atrophic. Another study using translimbal photocoagulation with a diode laser (with laser settings of 0.7 seconds and 0.4 Watts) that was aimed at either the TM and episcleral veins or only the TM of Wistar rats yielded an elevated IOP and subsequent glaucomatous damage which included RGC loss and abnormal outflow channels in the anterior chamber [60]. Peak IOP was $49.0 \mathrm{mmHg}$ in the combination group and $34 \mathrm{mmHg}$ in the TM only group. IOP remained elevated for 3 weeks for both methods and there was axonal loss with both methods.

As mentioned previously, rats are easy and more economical to maintain, and a large number can be treated in one day by one person reducing the cost associated with additional personnel. However, similar to other induced animal models, the technique may need multiple sessions to achieve IOP elevation. Although IOP elevation is achieved, the response to induction of glaucoma may be inconsistent. The hypertonic saline model is likely to be the most consistent model but is technically difficult to perform and has mainly been used in Brown Norway rats. The IOP elevation in all these models is sustained for a period of 2-6 weeks.

(2) Mice. Other mouse models that have been developed to study PACG included those developed by photocoagulation of the episcleral vessels $[62,63,109]$ and episcleral vein cauterization [64]. These models exhibited elevated IOP for up to 4 weeks, loss of RGCs, and damage of RGC axons. Translimbal-photocoagulation-treated eyes of Black Swiss mice reached a maximum IOP of $39.6 \mathrm{mmHg}$ with IOP elevation being statistically significant compared with controls for up to 6 weeks [109], whereas in photocoagulation of episcleral- and limbal-vein-treated Albino CD1 mice eyes had doubling of their IOP within 4 hours [62]. IOP in these models remained stable through the second postoperative day $(27.6 \mathrm{mmHg})$ but returned to baseline after one week. At day fourteen after treatment, there was a $42 \%$ loss of RGCs. When C57BL/6J mice were treated in a similar fashion as described previously, during the first four weeks after laser treatment, the mean IOP was $20 \mathrm{mmHg}$ compared with $13 \mathrm{mmHg}$ before treatment. Two weeks after laser photocoagulation, the percentage of RCG lost in treated eyes of these mice with elevated IOP compared to untreated controls was about $17 \%$ and at 4 weeks, and the death rate was $22.4 \%$ [63]. Using fluorescent polystyrene microbeads injection in C57BL/ 6 mice as well, a consistent $30 \%$ elevation in IOP that persisted for more than 3 weeks was achieved using 1 single injection [56].

(3) Rabbits. Rabbit models for angle closure glaucoma were also created by either water loading $[65,66]$ or argon laser energy applied to the TM [67-69]. Both pigmented and albino rabbits were used in these studies. Although elevated IOP and buphthalmia were achieved in these animals, these models all had drawbacks. For example, in the water loading models, the damage produced included the whole eye and the IOP rise was of insufficient duration $(1 \mathrm{hr})$ and caused selective loss of RGCs. In the laser-induced glaucoma models, the IOP elevation lasted for a few weeks but it was hard to achieve a successful model because of the structure of the iridocorneal angle, which is different from that of humans. The longest IOP elevation was reported in the $\alpha$ chymotrypsin-injected models.

1.3. Primary Congenital Glaucoma. Primary Congenital Glaucoma (PCG) is an autosomal recessive disease caused 
by an abnormal development of the anterior chamber angle. PCG has been linked to several genetic loci. CYP1B1 and LTBP2 are the only genes in which mutations are currently known. However, the role that these genes play in the pathophysiology of PCG and development of the anterior chamber is not known. An assortment of spontaneous glaucoma models has been described in different animal species (Table 1). These included rabbits, dogs, monkeys, mice, rats, cats, and albino quails. The study of these models has provided valuable information on the pathophysiology of glaucoma as it relates to changes in the anterior chamber angle, the $\mathrm{ON}$, and the retina but the mechanisms leading to these changes are still elusive.

1.3.1. Rabbits. Spontaneous Congenital Glaucoma in rabbits was first described in 1886 by Schloesser [70]. However, research with this model did not advance until the 1960s when Kolker et al. [73], Hanna et al. [72], and Fox et al. [71] described a group of albino New Zealand rabbits that spontaneously exhibited congenital abnormalities in the development of the anterior chamber. The abnormalities included loss or compression of the iris pillars (pectinate ligaments) and posterior displacement or poor development of the aqueous plexus. Additional findings include dilated or compressed intertrabecular spaces, disorganization of trabecular lamellae, decreased trabecular endothelial cells, and a loss of trabecular endothelial cell-to-cell associations. Others have reported the replacement of the angular meshwork (the trabecular meshwork-like structure in rabbits) with abundant extracellular matrix (ECM), basal-lamina like material, and unidentified round cells just beneath the aqueous plexus $[74,75]$. The anterior chamber angle dysgenesis in the rabbit appears to be secondary to an alteration in the differentiation and maintenance of the structural integrity of the angular meshwork. Some of these changes bear resemblance to angle changes seen in PCG in humans [72]. The interest in studying this model decreased in the 1990s when other genetic models of glaucoma became available.

Many studies have indicated that glaucoma in rabbits is most likely autosomal recessive with incomplete penetrance (semi lethal) [72]. It typically manifests in the first 6 months and is associated with variable IOP elevation, enlarged cloudy corneas, and elongated globes. The outflow facility is decreased, suggesting a defect in the outflow pathway, which correlates with the reported histological findings. RGC loss and cupping of the optic nerve were also observed in these rabbits. The phenotypic similarities between rabbits and human patients with congenital glaucoma include the age of onset, IOP elevation, and buphthalmia. In addition, the rabbit eye is also relatively large, which makes it a good model for eye research. However, there are some limitations that make this animal unsuitable for glaucoma research. These include differences in the structure of the trabecular meshwork and aqueous outflow pathways between the human and the rabbit making it difficult to make direct correlations between the developmental changes in the anterior chamber angle in both species. In addition, IOP levels in the buphthalmic rabbit were found to decrease with age. IOP was found to be comparable to normal (18$20 \mathrm{mmHg}$ ) until about 5 months of age, followed by intermittent elevation into the $30 \mathrm{mmHg}$ range. A decrease in outflow facility precedes the elevation of IOP. The IOP elevation among animals is variable $(20-30 \mathrm{mmHg})$ up to about 18 months of age; then it decreases to near the normal range between 24 and 48 months. The cause of IOP reduction to normal levels despite decreased outflow facility is unclear. The genome sequence of the rabbit was recently made available at http://www.ncbi.nlm.nih.gov/projects/genome/ guide/rabbit/. This will help identify the genetic defects that cause glaucoma. The biggest disadvantage of this model is its limited availability from commercial vendors. This model has recently been used to study the protein changes in the aqueous humor and has provided valuable information about proteomics and the histopathological changes seen in the anterior chamber of this rabbit, although a CYP1B1 mutation could not be identified in this model [110].

1.3.2. Rats. Congenital glaucoma in rats was first described in 1926 by Addison and How [76], and in 1974, Young et al. also reported a spontaneous occurrence of buphthalmos in a colony of WAG inbred rats [77]. The condition in the latter appeared to be inherited but no completely satisfactory mode of inheritance was given. These reports were followed by another one in 1975 by Heywood [78]. Recently, an RCS$\mathrm{rdy}^{-}$rat model that develops glaucoma spontaneously was also described $[78,79]$. The mutant animals had either a unilateral or bilateral enlargement of the globes with an IOP that ranged from 25 to $45 \mathrm{mmHg}$, as compared to control values of $12-16 \mathrm{mmHg}$. The IOP increased significantly with age to reach a value of $35 \pm 7.3$ at $12-18$ months of age. The animals also had decreased number of RGCs with age as well as atrophic ONHs. The anterior chamber was narrow and the iridocorneal angle was open. These rats were used in other studies in glaucoma research and yielded valuable information about RGC loss [111].

1.3.3. Cats. Feline glaucoma is a rare condition. It has been described in Burmese cats [80], domestic cats [6], and Siamese cats $[21,81]$. Examination of the Siamese cats revealed bilateral mild-to-moderate buphthalmos and moderate elevation in IOP, which was as high as $31.6 \mathrm{mmHg}$. Clinical features identified in these cats were similar to those seen in human PCG, though details such as IOP levels and clinical course were not described in these reports. Structurally, these cats had prominent, elongated ciliary processes, Haab's striae, and lens subluxation. Gonioscopic examination revealed open or slightly narrowed iridocorneal angles, with mild pectinate ligament dysplasia and sparse prominent iris processes. Histological examination of Burmese cat eyes revealed loss of RGC, corneal edema, and multifocal breaks in Descemet's membrane [112]. Similar to rabbits, the cat eyes are relatively larger making them attractive for use in glaucoma research. However, no further reports were published describing clinical, pathological, and genetic characterization of the disease in cats. 
1.3.4. Mice. A knockout model with Cyp1b1 has also been developed to simulate PCG where CYP1B1 mutations are the predominant cause of PCG in humans in some populations [82]. Cyp1b1-deficient mice exhibit abnormalities in their ocular drainage structure and TM that are similar to those reported in human PCG patients [83]. However, other studies generated a Cyp1b1-null mouse that revealed no evidence of glaucoma, and the animals were not blind [84]. A mouse model with mutations in both Cyp1b1 and Tyr (tyrosinase) was also developed. Studies using this model showed that the anterior segment developmental pathway involves a tyrosinase and that the Tyr mutation modifies the phenotype associated with inheritance of mutant orthologs of Cyp1b1 and Foxc1, which both have been shown to cause PCG in humans [85]. Although results are contradictory, studies using this model could lead to understanding the abnormalities seen in the ocular drainage and the structure of the TM. Similar to the buphthalmic rabbit described previously, CYP1B1 mutation could be specific to the human PCG only which makes this model and other PCG animal models not suitable for studying the genetics in this disease.

1.3.5. Albino Quails. The albino quail model of glaucoma (al mutant) was described in 1986 by Takatsuji et al. [86]. The al mutation is sex-linked semilethal recessive of known penetrance. The gene mutation has not been described. The bird exhibited enlargement of the eye, RGC degeneration, cupping of the optic disc, and cataract with retinal histopathological features similar to those in animals with experimentally induced or spontaneous glaucoma. Loss of RGCs was similar to human [87]. The al mutant quails show significantly higher IOP at 6 months of age. The iridocorneal angle is initially open but eventually closes in later stages of the disease [86]. The mutant bird would be a good model to study glaucoma, as it is easy to maintain and to handle in a laboratory. However, the cornea of these birds is very small and IOP measurement may be challenging, although a tonopen can be used. In addition, availability of the albino quail is also limited.

\subsection{Other Types of Glaucoma}

1.4.1. Normal Tension Glaucoma. Normal Pressure or Normal Tension Glaucoma (NTG) is a condition where the clinical features are largely identical to those seen in POAG except the IOP, which, in affected patients, is below the statistically normal upper limit $(21 \mathrm{mmHg})$. The pathophysiology of RGC degeneration and ON damage in NTG remains unclear. A number of factors have been implicated as potential mechanisms of RGC degeneration. Some of these include poor blood flow to the $\mathrm{ON}$, genetic mutations, and vascular spasm [113-115]. To explore the possible pathways of RGC degeneration, genetically modified mice with normal IOP have been utilized as models of NTG as described below.

Because glutamate excitotoxicity and oxidative stress have been implicated in RGC death, mice deficient in the glutamate transporter genes Glast or Eaac1 have been developed as models for normal tension glaucoma. These mice demonstrate RGC and ON degeneration without IOP elevation [88] suggesting that these transporters play important roles in preventing RGC degeneration by keeping the extracellular glutamate concentration below the neurotoxic level and maintaining the glutathione levels in Müller cells by synthesizing and transporting glutamate into the cells. Glutamate is the substrate for glutathione synthesis. This model was used to investigate ASK1 deficiency on neural cell death [116]. ASK1 is a mitogen-activated protein kinase (MAPK) kinase kinase and has an important role in stress-induced RGC apoptosis. The authors found that loss of ASK1 had no effects on the production of glutathione or malondialdehyde in the retina or on IOP. Tumor-necrosis-factor-(TNF-) induced activation of p38 MAPK and the production of inducible nitric oxide synthase were suppressed also in ASK1-deficient Müller glial cells and RGCs which suggested that ASK1 activation is involved in NTG.

1.4.2. Autoimmune Glaucoma. Several reports have suggested that an autoimmune response is one possible mechanism of RGC degeneration in normal pressure glaucoma [117]. To test this hypothesis, some studies have examined serum samples from glaucoma patients to look for autoantibodies and have found increased levels of heat shock protein 27 (HSP27) and heat shock protein 60 (HSP60). HSP27 and HSP60 immunization in the Lewis rat induced RGC degeneration and axonal loss 1-4 months later in a pattern similar to human glaucoma [89], suggesting the role of these proteins in the development of glaucoma. The models also showed IOP-independent RGC loss and changes in serum antibody patterns [90]. Experimental autoimmune glaucoma offers a valuable tool to examine the diverse roles of the immune system in glaucoma. It may also facilitate the identification of treatment strategies to prevent pressureindependent RGC degeneration as it may occur in select patients with glaucoma. However, depending on the animal used, limitations can be encountered such as the size of the eye, the cost of the animal, and the anatomical similarities of the animal's eye to that of the human.

1.4.3. Pigmentary Glaucoma. The DBA/2J mouse, which develops a progressive increase of IOP, was recognized in 1978 [118]. The glaucoma in this strain is caused by iris abnormalities related to recessive mutations in two genes, glycosylated protein $\mathrm{nmb}(\mathrm{Gpnmb})$ and tyrosinase-related protein 1 (Tyrp1) [91, 92]. Both mutations show incomplete penetrance. Therefore, only about $70 \%$ of animals develop glaucoma [93]. The mechanism of glaucoma is related to iris atrophy, pigment dispersion, and development of peripheral anterior synechiae leading to angle closure. IOP elevation in the DBA/2J mouse is seen at 8 months of age and remains until death. The mouse develops pigment dispersion which precedes iris atrophy, anterior synechiae, and elevated IOP. These changes are accompanied by retinal and ON changes consistent with glaucoma. The disease progresses with increasing age similar to glaucoma in humans $[92,118]$. However, there appears to be a closed angle component to 
the glaucoma unlike in humans where pigmentary glaucoma is of an open angle form. Although this model has been extensively studied, there is one limitation that makes this model not ideal for studying glaucoma: The elevated IOP phenotype is not primary, but secondary due to the systemic pigment dispersion syndrome with the associated mutations in the Gpnmb and Tyrp1 loci [91, 92]. In addition, mice with spontaneous glaucoma other than the DBA/2J, such as the $\mathrm{DBA} / 2$ and $\mathrm{DBA} / 2 \mathrm{NNia}$, are difficult to obtain commercially.

\section{Conclusion}

This paper describes most of the animal models utilized in glaucoma research to date. These animal models have provided valuable information about certain aspects of the disease process but the search for models that address knowledge gaps in specific forms of glaucoma must continue. The validity of each of these models depends upon the degree of similarity to the human condition as well as considerations of the model being economical and practical. Since the mechanisms of glaucoma differ among animal models, data obtained from a particular model should not be generalized and should be interpreted within the context of that model. The animal model used should be selected based on the experimental needs and the hypothesis being tested. For example, genetically induced models might be preferable to investigate the effects of elevated IOP on the ocular tissues over prolonged periods without the superimposed effects of experimental procedures or inflammation whereas spontaneously occurring large animal models such as monkeys, dogs, rabbit, and the recently described pig [8] offer a unique opportunity to collect data by using instrumentation identical to that used in human patients with glaucoma. Experimentally induced models have the advantage of studying some of the changes in glaucoma over a short period of time. However, sophisticated equipment and trained personnel to induce glaucoma are often needed. In addition, glaucoma induction can be somewhat unpredictable. These models may be useful in testing responses to medications. It is likely that genetic models developed to address specific hypotheses will provide valuable information on the pathophysiology of the various types and aspects of glaucoma and potentially lead to the discovery of new therapeutic targets.

\section{References}

[1] H. Quigley and A. T. Broman, "The number of people with glaucoma worldwide in 2010 and 2020," British Journal of Ophthalmology, vol. 90, no. 3, pp. 262-267, 2006.

[2] B. Thylefors, A. D. Négrel, R. Pararajasegaram, and K. Y. Dadzie, "Available data on blindness (update 1994)," Ophthalmic epidemiology, vol. 2, no. 1, pp. 5-39, 1995.

[3] K. N. Gelatt, “Animal models for glaucoma," Investigative Ophthalmology and Visual Science, vol. 16, no. 7, pp. 592-596, 1977.

[4] E. Vecino, "Animal models in the study of the glaucoma: past, present and future," Archivos de la Sociedad Espanola de Oftalmologia, vol. 83, no. 9, pp. 517-519, 2008.
[5] C. A. Rasmussen and P. L. Kaufman, "Primate glaucoma models," Journal of Glaucoma, vol. 14, no. 4, pp. 311-314, 2005.

[6] D. E. Brooks, "Glaucoma in the dog and cat," Veterinary Clinics of North America-Small Animal Practice, vol. 20, no. 3, pp. 775-797, 1990.

[7] U. Dietrich, "Feline glaucomas," Clinical Techniques in Small Animal Practice, vol. 20, no. 2, pp. 108-116, 2005.

[8] J. Ruiz-Ederra, M. García, M. Hernández et al., “The pig eye as a novel model of glaucoma," Experimental Eye Research, vol. 81, no. 5, pp. 561-569, 2005.

[9] I. H. Pang and A. F. Clark, "Rodent models for glaucoma retinopathy and optic neuropathy," Journal of Glaucoma, vol. 16, no. 5, pp. 483-505, 2007.

[10] H. A. Quigley, "Open-angle glaucoma," New England Journal of Medicine, vol. 328, no. 15, pp. 1097-1106, 1993.

[11] W. W. Dawson, D. E. Brooks, G. M. Hope et al., "Primary open angle glaucomas in the rhesus monkey," British Journal of Ophthalmology, vol. 77, no. 5, pp. 302-310, 1993.

[12] D. Gaasterland and C. Kupfer, "Experimental glaucoma in the rhesus monkey," Investigative Ophthalmology, vol. 13, no. 6, pp. 455-457, 1974.

[13] H. A. Quigley, R. M. Sanchez, and G. R. Dunkelberger, "Chronic glaucoma selectively damages large optic nerve fibers," Investigative Ophthalmology and Visual Science, vol. 28, no. 6, pp. 913-920, 1987.

[14] Y. Glovinsky, H. A. Quigley, and G. R. Dunkelberger, "Retinal ganglion cell loss is size dependent in experimental glaucoma," Investigative Ophthalmology and Visual Science, vol. 32, no. 3, pp. 484-491, 1991.

[15] Y. Glovinsky, H. A. Quigley, and M. E. Pease, "Foveal ganglion cell loss is size dependent in experimental glaucoma," Investigative Ophthalmology and Visual Science, vol. 34, no. 2, pp. 395-400, 1993.

[16] H. A. Quigley and R. M. Hohman, "Laser energy levels for trabecular meshwork damage in the primate eye," Investigative Ophthalmology \& Visual Science, vol. 24, no. 9, pp. 13051307, 1983.

[17] C. B. Toris, G. L. Zhan, Y. L. Wang et al., "Aqueous humor dynamics in monkeys with laser-induced glaucoma," Journal of Ocular Pharmacology and Therapeutics, vol. 16, no. 1, pp. 19-27, 2000.

[18] A. J. Weber and D. Zelenak, "Experimental glaucoma in the primate induced by latex microspheres," Journal of Neuroscience Methods, vol. 111, no. 1, pp. 39-48, 2001.

[19] H. A. Quigley and E. M. Addicks, "Chronic experimental glaucoma in primates. I. Production of elevated intraocular pressure by anterior chamber injection of autologous ghost red blood cells," Investigative Ophthalmology and Visual Science, vol. 19, no. 2, pp. 126-136, 1980.

[20] H. A. Quigley and E. M. Addicks, "Chronic experimental glaucoma in primates. II. Effect of extended intraocular pressure elevation on optic nerve head and axonal transport," Investigative Ophthalmology and Visual Science, vol. 19, no. 2, pp. 137-152, 1980.

[21] K. N. Gelatt, G. G. Gum, and R. M. Gwin, "Animal model of human disease. Primary open angle glaucoma. Inherited primary open angle glaucoma in the beagle," American Journal of Pathology, vol. 102, no. 2, pp. 292-295, 1981.

[22] J. Kuchtey, L. M. Olson, T. Rinkoski et al., "Mapping of the disease locus and identification of ADAMTS10 as a candidate gene in a canine model of primary open angle Glaucoma," PLoS Genetics, vol. 7, no. 2, Article ID e1001306, 2011. 
[23] V. Senatorov, I. Malyukova, R. Fariss et al., "Expression of mutated mouse myocilin induces open-angle glaucoma in transgenic mice," Journal of Neuroscience, vol. 26, no. 46, pp. 11903-11914, 2006.

[24] Y. Zhou, O. Grinchuk, and S. I. Tomarev, "Transgenic mice expressing the Tyr437His mutant of human myocilin protein develop glaucoma," Investigative Ophthalmology and Visual Science, vol. 49, no. 5, pp. 1932-1939, 2008.

[25] F. Mabuchi et al., "Optic nerve damage in mice with a targeted type I collagen mutation," Investigative Ophthalmology \& Visual Science, vol. 45, no. 6, pp. 1841-1845, 2004.

[26] M. Aihara, J. D. Lindsey, and R. N. Weinreb, "Ocular hypertension in mice with a targeted type I collagen mutation," Investigative Ophthalmology and Visual Science, vol. 44, no. 4, pp. 1581-1585, 2003.

[27] K. Sawaguchi, Y. Nakamura, Y. Nakamura, H. Sakai, and S. Sawaguchi, "Myocilin gene expression in the trabecular meshwork of rats in a steroid-induced ocular hypertension model," Ophthalmic Research, vol. 37, no. 5, pp. 235-242, 2005.

[28] S. W. John, R. S. Smith, B. D. Perkins et al., "Characterization of the zebrafish bug eye mutation, exploring a genetic model for pressure-induced retinal cell death," Investigative Ophthalmology \& Visual Science, vol. 44, E- Abstract 1125, 2003.

[29] J. M. Stujenske, J. E. Dowling, and F. Emran, "The bugeye mutant zebrafish exhibits visual deficits that arise with the onset of an enlarged eye phenotype," Investigative Ophthalmology \& Visual Science, vol. 52, no. 7, pp. 4200-4207, 2011.

[30] K. N. Veth, J. R. Willer, R. F. Collery et al., "Mutations in zebrafish Lrp2 result in adult-onset ocular pathogenesis that models myopia and other risk factors for glaucoma," PLoS Genetics, vol. 7, no. 2, Article ID e1001310, 2011.

[31] J. M. Skarie and B. A. Link, "The Primary open-angle glaucoma gene WDR36 functions in ribosomal RNA processing and interacts with the p53 stress-response pathway," Human Molecular Genetics, vol. 17, no. 16, pp. 2474-2485, 2008.

[32] L. Bonomi, S. Perfetti, and E. Noya, "Experimental corticosteroid ocular hypertension in the rabbit," Albrecht von Graefes Archiv fur Klinische und Experimentelle Ophthalmologie, vol. 209, no. 2, pp. 73-82, 1978.

[33] U. Ticho, M. Lahav, S. Berkowitz, and P. Yoffe, "Ocular changes in rabbits with corticosteroid-induced ocular hypertension," British Journal of Ophthalmology, vol. 63, no. 9, pp. 646-650, 1979.

[34] D. C. Wood, D. Sweet, I. Contaxis, and J. Van Dolah, "Response of rabbits to corticosteroids. II. Influence of topical therapy on lens, aqueous humor, serum and urine composition," American Journal of Ophthalmology, vol. 63, no. 4, pp. 849-856, 1967.

[35] P. Chee and D. I. Hamasaki, "The basis for chymotrypsininduced glaucoma.," Archives of Ophthalmology, vol. 85, no. 1, pp. 103-106, 1971.

[36] M. Best, A. Z. Rabinovitz, and S. Masket, "Experimental alphachymotrypsin glaucoma," Annals of Ophthalmology, vol. 7, no. 6, pp. 803-810, 1975.

[37] S. Lessell and T. Kuwabara, "Experimental alpha-chymotrypsin glaucoma.," Archives of Ophthalmology, vol. 81, no. 6, pp. 853-864, 1969.

[38] O. A. Candia, R. Gerometta, J. C. Millar, and S. M. Podos, "Suppression of corticosteroid-induced ocular hypertension in sheep by anecortave," Archives of Ophthalmology, vol. 128, no. 3, pp. 338-343, 2010.
[39] R. Gerometta, M. G. Spiga, T. Borrás, and O. A. Candia, "Treatment of sheep steroid-induced ocular hypertension with a glucocorticoid-inducible MMP1 gene therapy virus," Investigative Ophthalmology and Visual Science, vol. 51, no. 6, pp. 3042-3048, 2010.

[40] R. Gerometta, S. M. Podos, J. Danias, and O. A. Candia, "Steroid-induced ocular hypertension in normal sheep," Investigative Ophthalmology and Visual Science, vol. 50, no. 2, pp. 669-673, 2009.

[41] O. Y. Tektas, C. M. Hammer, J. Danias et al., "Morphologic changes in the outflow pathways of bovine eyes treated with corticosteroids," Investigative Ophthalmology and Visual Science, vol. 51, no. 8, pp. 4060-4066, 2010.

[42] R. Gerometta, S. M. Podos, O. A. Candia et al., "Steroidinduced ocular hypertension in normal cattle," Archives of Ophthalmology, vol. 122, no. 10, pp. 1492-1497, 2004.

[43] J. K. Lauber, "Light-induced avain glaucoma as an animal model for human primary glaucoma," Journal of Ocular Pharmacology, vol. 3, no. 1, pp. 77-100, 1987.

[44] A. Kinnear, J. K. Lauber, and T. A. S. Boyd, "Genesis of light induced avian glaucoma," Investigative Ophthalmology, vol. 13, no. 11, pp. 872-875, 1974.

[45] J. K. Lauber, M. A. McLaughlin, and G. C. Y. Chiou, "Timolol and pilocarpine are hypotensive in light-induced avian glaucoma," Canadian Journal of Ophthalmology, vol. 20, no. 4, pp. 147-152, 1985.

[46] C. L. Martin and M. Wyman, "Glaucoma in the Basset Hound.," Journal of the American Veterinary Medical Association, vol. 153, no. 10, pp. 1320-1327, 1968.

[47] H. M. Whigham, D. E. Brooks, S. E. Andrew, K. N. Gelatt, D. T. Strubbe, and D. J. Biros, "Treatment of equine glaucoma by transscleral neodymium:yttrium aluminum garnet laser cyclophotocoagulation: a retrospective study of 23 eyes of 16 horses," Veterinary Ophthalmology, vol. 2, no. 4, pp. 243-250, 1999.

[48] L. G. Lovekin, "Primary Glaucoma in Dogs," Journal of the American Veterinary Medical Association, vol. 145, pp. 10811091, 1964.

[49] W. G. Magrane, "Canine glaucoma. II. Primary classification," Journal of the American Veterinary Medical Association, vol. 131, no. 8, pp. 372-374, 1957.

[50] M. Wyman and K. Ketring, "Congenital glaucoma in the basset hound: a biologic model," Transactions of the American Academy of Ophthalmology and Otolaryngology, vol. 81, no. 4, pp. OP645-OP652, 1976.

[51] K. N. Gelatt et al., "Glaucoma in the beagle," Transactions of the American Academy of Ophthalmology and Otolaryngology, vol. 81, no. 4, pp. OP636-OP644, 1976.

[52] K. N. Gelatt et al., "Clinical manifestations of inherited glaucoma in the beagle," Investigative Ophthalmology \& Visual Science, vol. 16, no. 12, pp. 1135-1142, 1977.

[53] S. Reinstein, A. Rankin, and R. Allbaugh, "Canine glaucoma: pathophysiology and diagnosis," Compendium: Continuing Education For Veterinarians, vol. 31, no. 10, pp. 450-453, 2009.

[54] A. W. De Kater, R. Smyth, R. C. Rosenquist, and D. L. Epstein, "The Slate turkey: a model for secondary angle closure glaucoma," Investigative Ophthalmology and Visual Science, vol. 27, no. 12, pp. 1751-1754, 1986.

[55] J. C. Morrison, C. G. Moore, L. M. H. Deppmeier, B. G. Gold, C. K. Meshul, and E. C. Johnson, "A rat model of chronic pressure-induced optic nerve damage," Experimental Eye Research, vol. 64, no. 1, pp. 85-96, 1997. 
[56] R. M. Sappington et al., "The microbead occlusion model: a paradigm for induced ocular hypertension in rats and mice," Investigative Ophthalmology \& Visual Science, vol. 51, no. 1, pp. 207-216, 2009.

[57] M. C. Moreno, H. J. Aldana Marcos, J. O. Croxatto et al., "A new experimental model of glaucoma in rats through intracameral injections of hyaluronic acid," Experimental Eye Research, vol. 81, no. 1, pp. 71-80, 2005.

[58] S. R. Shareef, E. Garcia-Valenzuela, A. Salierno, J. Walsh, and S. C. Sharma, "Chronic ocular hypertension following episcleral venous occlusion in rats," Experimental Eye Research, vol. 61, no. 3, pp. 379-382, 1995.

[59] S. Yu, T. Tanabe, and N. Yoshimura, "A rat model of glaucoma induced by episcleral vein ligation," Experimental Eye Research, vol. 83, no. 4, pp. 758-770, 2006.

[60] H. Levkovitch-Verbin, H. A. Quigley, K. R. G. Martin, D. Valenta, L. A. Baumrind, and M. E. Pease, "Translimbal laser photocoagulation to the trabecular meshwork as a model of glaucoma in rats," Investigative Ophthalmology and Visual Science, vol. 43, no. 2, pp. 402-410, 2002.

[61] K. Fujikawa, T. Iwata, K. Inoue et al., "VAV2 and VAV3 as candidate disease genes for spontaneous glaucoma in mice and humans," PLoS One, vol. 5, no. 2, Article ID e9050, 2010.

[62] C. T. Fu and D. Sretavan, "Laser-induced ocular hypertension in albino CD-1 mice," Investigative Ophthalmology and Visual Science, vol. 51, no. 2, pp. 980-990, 2010.

[63] R. L. Gross, J. Ji, P. Chang et al., "A mouse model of elevated intraocular pressure: retina and optic nerve findings," Transactions of the American Ophthalmological Society, vol. 101, pp. 163-171, 2003.

[64] J. Ruiz-Ederra and A. S. Verkman, "Mouse model of sustained elevation in intraocular pressure produced by episcleral vein occlusion," Experimental Eye Research, vol. 82, no. 5, pp. 879-884, 2006.

[65] T. O. McDonald, J. W. Hodges, A. R. Borgmann, and F. E. Leaders, "The water-loading test in rabbits. A method to detect potential ocular hypotensive drugs.", Archives of Ophthalmology, vol. 82, no. 3, pp. 381-384, 1969.

[66] R. M. Thorpe and A. E. Kolker, "A tonographic study of water loading in rabbits.," Archives of Ophthalmology, vol. 77, no. 2, pp. 238-243, 1967.

[67] T. Gherezghiher, W. F. March, R. E. Nordquist, and M. C. Koss, "Laser-induced glaucoma in rabbits," Experimental Eye Research, vol. 43, no. 6, pp. 885-894, 1986.

[68] B. Johnson, P. House, W. Morgan, X. Sun, and D. Y. Yu, "Developing laser-induced glaucoma in rabbits," Australian and New Zealand Journal of Ophthalmology, vol. 27, no. 3-4, pp. 180-183, 1999.

[69] R. J. Seidehamel and K. W. Dungan, "Characteristics and pharmacologic utility of an intraocular pressure (IOP) model in unanesthetized rabbits," Investigative Ophthalmology, vol. 13, no. 4, pp. 319-322, 1974.

[70] C. V. Schloesser, "Acutes Secundar-Glaucom beim Kaninchen," Zeitschrift für Vergleichende Augenheilkunde, vol. 35, no. 4, pp. 232-233, 1886.

[71] R. R. Fox, D. D. Crary, E. J. Babino, and B. Sheppard, "Buphthalmia in the rabbit: pleiotropic effects of the (bu) gene and a possible explanation of mode of gene action," Journal of Heredity, vol. 60, no. 4, pp. 206-212, 1969.

[72] B. L. Hanna, P. B. Sawin, and L. B. Sheppard, "Recessive buphthalmos in the rabbit," Genetics, vol. 47, pp. 519-529, 1962.

[73] A. E. Kolker, R. A. Moses, M. A. Constant, and B. Becker, "The development of glaucoma in rabbits," Investigative ophthalmology, vol. 2, pp. 316-321, 1963.
[74] A. Ueno, A. Tawara, T. Kubota, Y. Ohnishi, H. Inomata, and A. S. Solomon, "Histopathological changes in iridocorneal angle of inherited glaucoma in rabbits," Graefe's Archive for Clinical and Experimental Ophthalmology, vol. 237, no. 8, pp. 654-660, 1999.

[75] P. A. Knepper, D. G. McLone, W. Goossens, T. Vanden Hoek, and R. G. Higbee, "Ultrastructural alterations in the aqueous outflow pathway of adult buphthalmic rabbits," Experimental Eye Research, vol. 52, no. 5, pp. 525-533, 1991.

[76] W. H. F. Addison and H. W. How, "Congenital hypertrophy of the eye in an albino rat," The Anatomical Record, vol. 32, no. 4, pp. 271-277, 1926.

[77] C. Young, M. F. W. Festing, and K. C. Barnett, "Buphthalmos (congenital glaucoma) in the rat," Laboratory Animals, vol. 8, no. 1, pp. 21-31, 1974.

[78] R. Heywood, "Glaucoma in the rat," British Veterinary Journal, vol. 131, no. 2, pp. 213-221, 1975.

[79] R. Naskar and S. Thanos, "Retinal gene profiling in a hereditary rodent model of elevated intraocular pressure," Molecular Vision, vol. 12, pp. 1199-1210, 2006.

[80] E. C. G. M. Hampson, R. I. E. Smith, and M. E. Bernays, "Primary glaucoma in Burmese cats," Australian Veterinary Journal, vol. 80, no. 11, pp. 672-680, 2002.

[81] G. J. McLellan, D. M. Betts, K. Sigle, and S. Grozdanic, "Congenital glaucoma in the siamese cat-a novel spontaneous animal model for glaucoma research," Investigative Ophthalmology \& Visual Science, vol. 46: E-Abstract 134, 1995.

[82] B. A. Bejjani, R. A. Lewis, K. F. Tomey et al., "Mutations in CYP1B1, the gene for cytochrome P4501B1, are the predominant cause of primary congenital glaucoma in Saudi Arabia," American Journal of Human Genetics, vol. 62, no. 2, pp. 325333, 1998.

[83] M. Sarfarazi and I. Stoilov, "Molecular genetics of primary congenital glaucoma," Eye, vol. 14, no. 3B, pp. 422-428, 2000.

[84] J. T. M. Buters, S. Sakai, T. Richter et al., "Cytochrome P450 CYP1B1 determines susceptibility to 7,12-dimethylbenz[a] anthracene-induced lymphomas," Proceedings of the National Academy of Sciences of the United States of America, vol. 96, no. 5, pp. 1977-1982, 1999.

[85] R. T. Libby, R. S. Smith, O. V. Savinova et al., "Modification of ocular defects in mouse developmental glaucoma models by tyrosinase," Science, vol. 299, no. 5612, pp. 1578-1581, 2003.

[86] K. Takatsuji et al., "Animal model of closed angle glaucoma in albino mutant quails," Investigative Ophthalmology \& Visual Science, vol. 27, no. 3, pp. 396-400, 1986.

[87] K. Takatsuji et al., "Selective loss of retinal ganglion cells in albino avian glaucoma," Investigative Ophthalmology \& Visual Science, vol. 29, no. 6, pp. 901-909, 1988.

[88] T. Harada, C. Harada, K. Nakamura et al., "The potential role of glutamate transporters in the pathogenesis of normal tension glaucoma," Journal of Clinical Investigation, vol. 117, no. 7, pp. 1763-1770, 2007.

[89] M. B. Wax, G. Tezel, J. Yang et al., "Induced autoimmunity to heat shock proteins elicits glaucomatous loss of retinal ganglion cell neurons via activated T-cell-derived fas-ligand," Journal of Neuroscience, vol. 28, no. 46, pp. 12085-12096, 2008.

[90] S. C. Joachim et al., "Complex antibody profile changes in an experimental autoimmune glaucoma animal model," Investigative Ophthalmology \& Visual Science, vol. 50, no. 10, pp. 4734-4742, 2009.

[91] B. Chang, R. S. Smith, N. L. Hawes et al., "Interacting loci cause severe iris atrophy and glaucoma in DBA/2J mice," Nature Genetics, vol. 21, no. 4, pp. 405-409, 1999. 
[92] M. G. Anderson, R. S. Smith, N. L. Hawes et al., "Mutations in genes encoding melanosomal proteins cause pigmentary glaucoma in DBA/2J mice," Nature Genetics, vol. 30, no. 1, pp. 81-85, 2002.

[93] R. T. Libby, M. G. Anderson, I. H. Pang et al., "Inherited glaucoma in DBA/2J mice: pertinent disease features for studying the neurodegeneration," Visual Neuroscience, vol. 22, no. 5, pp. 637-648, 2005.

[94] B. J. Fan, D. Y. Wang, D. S. C. Lam, and C. P. Pang, "Gene mapping for primary open angle glaucoma," Clinical Biochemistry, vol. 39, no. 3, pp. 249-258, 2006.

[95] H. A. Quigley, R. W. Flower, E. M. Addicks, and D. S. McLeod, "The mechanism of optic nerve damage in experimental acute intraocular pressure elevation," Investigative Ophthalmology and Visual Science, vol. 19, no. 5, pp. 505-517, 1980.

[96] J. H. Fingert, E. M. Stone, V. C. Sheffield, and W. L. M. Alward, "Myocilin glaucoma," Survey of Ophthalmology, vol. 47, no. 6, pp. 547-561, 2002.

[97] E. Van Der Zypen, "Experimental morphological study on structure and function of the filtration angle of the rat eye," Ophthalmologica, vol. 174, no. 5, pp. 285-298, 1977.

[98] T. Daimon, M. Kazama, Y. Miyajima, and M. Nakano, "Immunocytochemical localization of thrombomodulin in the aqueous humor passage of the rat eye," Histochemistry and Cell Biology, vol. 108, no. 2, pp. 121-131, 1997.

[99] Ch. Reme, B. Aeberhard, and U. Urner, "The development of the chamber angle in the rat eye. Morphological characteristics of developmental stages," Graefe's Archive for Clinical and Experimental Ophthalmology, vol. 220, no. 3, pp. 139153, 1983.

[100] P. Nucci, G. Tredici, M. P. Manitto, G. Pizzini, and R. Brancato, "Neuron-specific enolase and embryology of the trabecular meshwork of the rat eye: an immunohistochemical study," International Journal of Biological Markers, vol. 7, no. 4, pp. 253-255, 1992.

[101] I. H. Pang, W. H. Wang, and A. F. Clark, "Acute effects of glaucoma medications on rat intraocular pressure," Experimental Eye Research, vol. 80, no. 2, pp. 207-214, 2005.

[102] C. McMahon, E. V. Semina, and B. A. Link, "Using zebrafish to study the complex genetics of glaucoma," Comparative Biochemistry and Physiology C, vol. 138, no. 3, pp. 343-350, 2004.

[103] B. A. Link, M. P. Gray, R. S. Smith, and S. W. M. John, "Intraocular pressure in zebrafish: comparison of inbred strains and identification of a reduced melanin mutant with raised IOP," Investigative Ophthalmology and Visual Science, vol. 45, no. 12, pp. 4415-4422, 2004.

[104] B. A. Barut and L. I. Zon, "Realizing the potential of zebrafish as a model for human disease," Physiological Genomics, vol. 2000, no. 2, pp. 49-51, 2000.

[105] P. Goldsmith, H. Baier, and W. A. Harris, "Two zebrafish mutants, ebony and ivory, uncover benefits of neighborhood on photoreceptor survival," Journal of Neurobiology, vol. 57, no. 3, pp. 235-245, 2003.

[106] R. Jones and D. J. Rhee, "Corticosteroid-induced ocular hypertension and glaucoma: a brief review and update of the literature," Current Opinion in Ophthalmology, vol. 17, no. 2, pp. 163-167, 2006.

[107] J. P. Kersey and D. C. Broadway, "Corticosteroid-induced glaucoma: a review of the literature," Eye, vol. 20, no. 4, pp. 407-416, 2006.

[108] A. L. Coleman, "Glaucoma," Lancet, vol. 354, no. 9192, pp. 1803-1810, 1999.
[109] M. Aihara, J. D. Lindsey, and R. N. Weinreb, "Experimental mouse ocular hypertension: establishment of the model," Investigative Ophthalmology and Visual Science, vol. 44, no. 10, pp. 4314-4320, 2003.

[110] D. P. Edward and R. Bouhenni, "Anterior segment alterations and comparative aqueous humor proteomics in the buphthalmic rabbit," Transactions of the American Ophthalmological Society, vol. 109, pp. 66-114, 2011.

[111] Z. Gatzioufas, P. Charalambous, B. Seitz et al., "Cholinergic inhibition by botulinum toxin in a rat model of congenital glaucoma raises intraocular pressure," British Journal of Ophthalmology, vol. 92, no. 6, pp. 826-831, 2008.

[112] S. Jacobi and R. R. Dubielzig, "Feline primary open angle glaucoma," Veterinary Ophthalmology, vol. 11, no. 3, pp. 162$165,2008$.

[113] M. B. Wax, "The case for autoimmunity in glaucoma," Experimental Eye Research, vol. 93, no. 2, pp. 187-190, 2010.

[114] J. Caprioli and A. L. Coleman, "Blood pressure, perfusion pressure, and glaucoma," American Journal of Ophthalmology, vol. 149, no. 5, pp. 704-712, 2010.

[115] M. B. Shields, "Normal-tension glaucoma: is it different from primary open-angle glaucoma?" Current Opinion in Ophthalmology, vol. 19, no. 2, pp. 85-88, 2008.

[116] C. Harada, K. Namekata, X. Guo et al., "ASK1 deficiency attenuates neural cell death in GLAST-deficient mice, a model of normal tension glaucoma," Cell Death and Differentiation, vol. 17, no. 11, pp. 1751-1759, 2010.

[117] I. Maruyama, M. Nakazawa, and H. Ohguro, "Autoimmune mechanisms in molecular pathology of glaucomatous optic neuropathy," Nippon Ganka Gakkai Zasshi, vol. 105, no. 4, pp. 205-212, 2001.

[118] S. W. John and al. et, "Essential iris atrophy, pigment dispersion, and glaucoma in DBA/2J mice," Investigative Ophthalmology \& Visual Science, vol. 39, no. 6, pp. 951-962, 1998. 


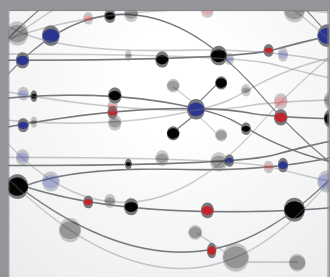

The Scientific World Journal
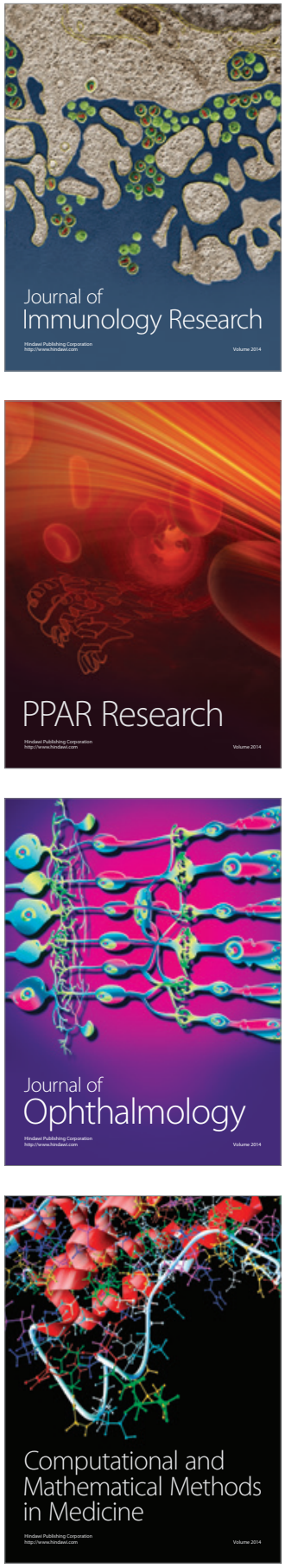

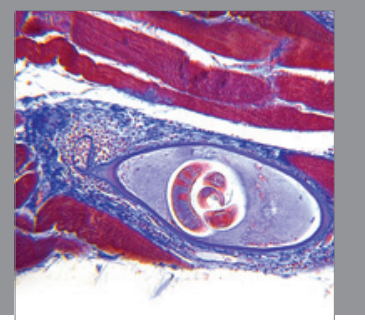

Gastroenterology

Research and Practice
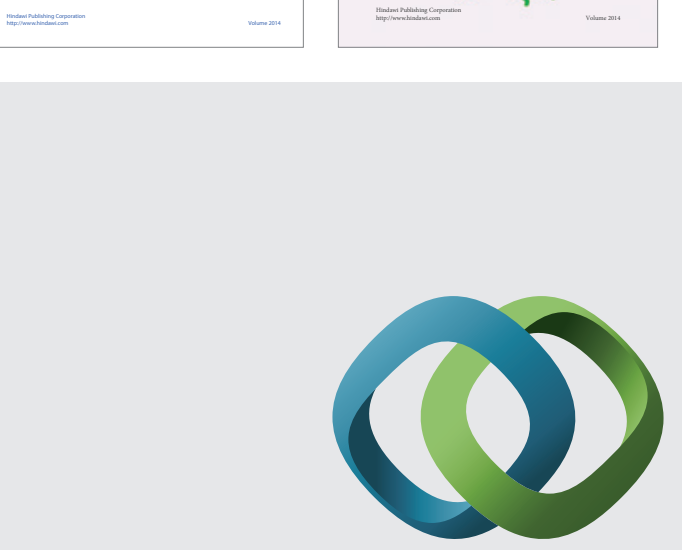

\section{Hindawi}

Submit your manuscripts at

http://www.hindawi.com
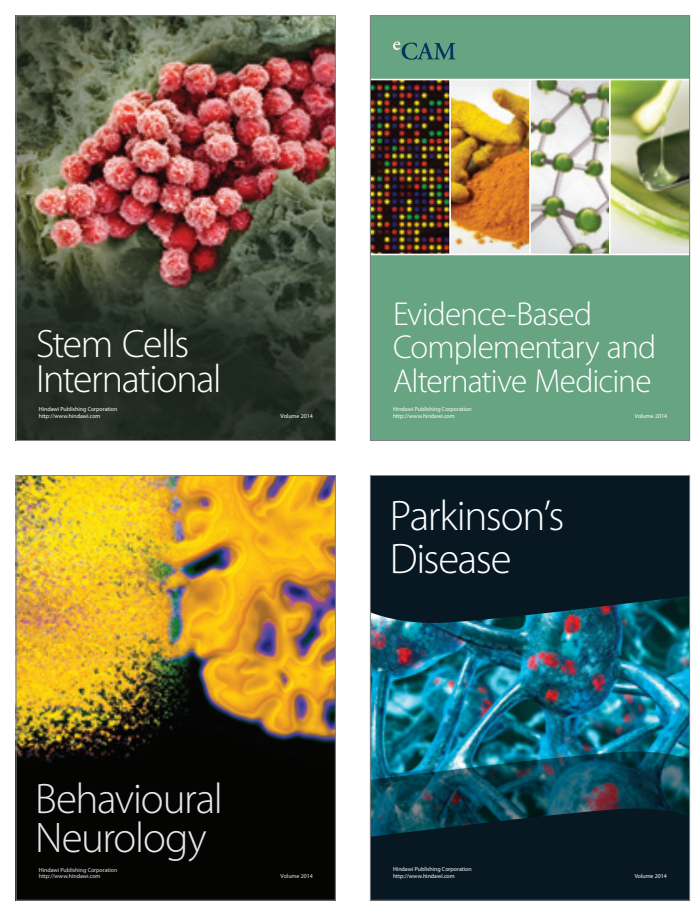

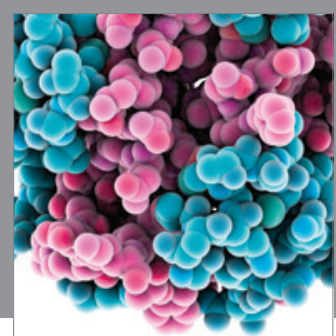

Journal of
Diabetes Research

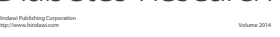

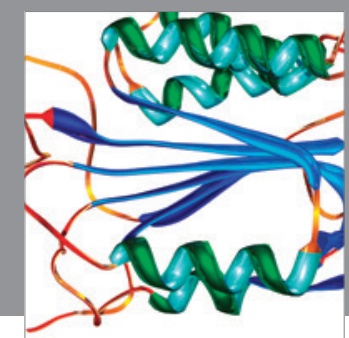

Disease Markers
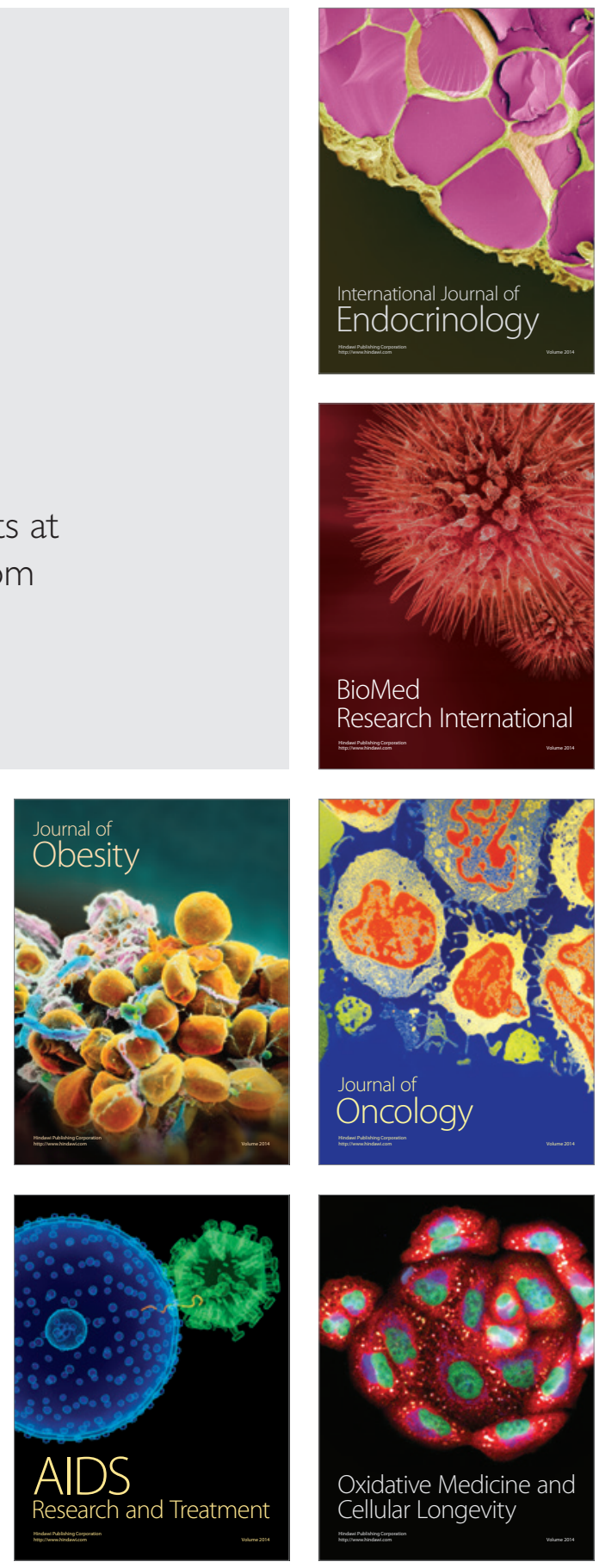